\title{
A conjecture by Leon Ehrenpreis about zeroes of exponential polynomials
}

\author{
Alain Yger \\ July 15, 2011
}

Dedicated to the memory of Leon Ehrenpreis.

\section{The conjecture, various formulations}

In [36], page 322, Leon Ehrenpreis formulated the following conjecture.

Conjecture 1.1 (original form, incorrect) If $F_{1}, \ldots, F_{N}$ are $N$ exponential polynomials in $n$ variables with purely imaginary algebraic frequencies, namely

$F_{j}\left(z_{1}, \ldots, z_{n}\right)=\sum_{k=0}^{M_{j}} b_{j k}(z) e^{i\left\langle\alpha_{j k}, z\right\rangle}, b_{j k} \in \mathbb{C}\left[X_{1}, \ldots, X_{n}\right], \alpha_{j k} \in \overline{\mathbb{Q}}^{n} \cap \mathbb{R}^{n}, j=1, \ldots, N$,

then the ideal $\left(F_{1}, \ldots, F_{N}\right)$ they generate in the Paley-Wiener algebra $\widehat{\mathcal{E}^{\prime}\left(\mathbb{R}^{n}\right)}$ is slowly decreasing respect to the Paley-Wiener weight $p(z)=\log |z|+|\operatorname{Im} z|$. As a consequence $^{1}$, this ideal is closed in $\widehat{\mathcal{E}^{\prime}\left(\mathbb{R}^{n}\right)}$. It coincides with the ideal $\left[I\left(F_{1}, \ldots, F_{N}\right)\right]_{\mathrm{loc}}$, which consists of elements in $\widehat{\mathcal{E}^{\prime}\left(\mathbb{R}^{n}\right)}$ that belong locally to the ideal generated by $F_{1}, \ldots, F_{N}$ in the algebra of entire functions in $n$ variables.

This conjecture, in a slightly modified form (see Conjecture 1.2), has been the inspiration for the joint work of C.A. Berenstein and the author since 1985. It is a challenging and fascinating question, one that is closely connected with other open questions in number theory and analytic geometry. In this note, I will point out many of these connections, detail some of the progress that has been made on the problem, and hopefully inspire others to continue the work.

\footnotetext{
${ }^{1}$ This follows from Theorem 11.2 in [36].
} 
As it stands, Conjecture 1.1 would imply, in the one variable setting, the following : if

$$
f(z)=\sum_{k=0}^{M} b_{k}(z) e^{i \alpha_{k} z}, \quad b_{k} \in \mathbb{C}[X], \quad \alpha_{k} \in \overline{\mathbb{Q}} \cap \mathbb{R}
$$

is an exponential polynomial in one variable with algebraic frequencies and all simple zeroes, then the ideal $\left(f, f^{\prime}\right)$ is a non proper ideal in $\widehat{\mathcal{E}^{\prime}(\mathbb{R})}$ which would imply

$$
|f(z)|+\left|f^{\prime}(z)\right| \geq c \frac{e^{-A|\operatorname{Im} z|}}{(1+|z|)^{p}}
$$

for some $c, A>0$ and $p \in \mathbb{N}$. Unfortunately, such an assertion is false if one does not set any condition of arithmetic nature on the polynomial coefficients $b_{k}$. Take for example

$$
f(z)=f_{\gamma}(z)=\sin (z-\gamma)-\sin (\sqrt{2}(z-\gamma)),
$$

where $2 \gamma / \pi$ has excellent approximations belonging to $(2 \mathbb{Z}+1) \oplus \sqrt{2}(2 \mathbb{Z}+1)$; then some zeroes of $f_{\gamma}$ of the form

$$
\frac{2 l \pi}{1-\sqrt{2}}, l \in \mathbb{Z}
$$

will approach extremely well other zeroes of $f_{\gamma}$ of the form

$$
\frac{2 \alpha+\left(2 l^{\prime}+1\right) \pi}{1+\sqrt{2}}, l^{\prime} \in \mathbb{Z}
$$

and thus the ideal $\left(f_{\gamma}, f_{\gamma}^{\prime}\right)$ fails to be closed in $\widehat{\mathcal{E}^{\prime}(\mathbb{R})}$. So Conjecture 1.1 needs to be reformulated as follows.

Conjecture 1.2 (revised form) If $F_{1}, \ldots, F_{N}$ are exponential polynomials in $n$ variables with both algebraic coefficients and purely imaginary algebraic frequencies, namely

$F_{j}\left(z_{1}, \ldots, z_{n}\right)=\sum_{k=0}^{M_{j}} b_{j k}(z) e^{i\left\langle\alpha_{j k}, z\right\rangle}, b_{j k} \in \overline{\mathbb{Q}}\left[X_{1}, \ldots, X_{n}\right], \alpha_{j k} \in \overline{\mathbb{Q}}^{n} \cap \mathbb{R}^{n}, j=1, \ldots, N$,

then the ideal $\left(F_{1}, \ldots, F_{N}\right)$ they generate in the Paley-Wiener algebra $\widehat{\mathcal{E}^{\prime}\left(\mathbb{R}^{n}\right)}$ is slowly decreasing respect to the Paley-Wiener weight $p(z)=\log |z|+|\operatorname{Im} z|$. As a consequence, this ideal is closed in $\widehat{\mathcal{E}^{\prime}\left(\mathbb{R}^{n}\right)}$, and thus coincides with the set of elements in $\widehat{\mathcal{E}^{\prime}\left(\mathbb{R}^{n}\right)}$ which belong locally to the ideal generated by $F_{1}, \ldots, F_{N}$ in the algebra of entire functions in $n$ variables. 
Such a conjecture appears to be stronger than the following one.

Conjecture 1.3 (weaker revised form) If $F_{1}, \ldots, F_{N}$ are exponential polynomials in $n$ variables as in Conjecture 1.2, namely

$F_{j}\left(z_{1}, \ldots, z_{n}\right)=\sum_{k=0}^{M_{j}} b_{j k}(z) e^{i\left\langle\alpha_{j k}, z\right\rangle}, b_{j k} \in \overline{\mathbb{Q}}\left[X_{1}, \ldots, X_{n}\right], \alpha_{j k} \in \overline{\mathbb{Q}}^{n} \cap \mathbb{R}^{n}, j=1, \ldots, N$

then the the closure of the ideal $\left(F_{1}, \ldots, F_{N}\right)$ they generate in the Paley-Wiener algebra $\widehat{\mathcal{E}^{\prime}\left(\mathbb{R}^{n}\right)}$ coincides with the set of elements in $\widehat{\mathcal{E}^{\prime}\left(\mathbb{R}^{n}\right)}$ which belong locally to the ideal generated by $F_{1}, \ldots, F_{N}$ in the algebra of entire functions in $n$ variables

The conjecture is equivalent to the assertion that the underlying system of differencedifferential equations $\mu_{1} * f=\cdots=\mu_{N} * f=0$ satisfies the spectral synthesis property.

With C.A. Berenstein, we have been developing since [16] a long-term joint research program originally devoted to various attempts to tackle Conjecture 1.2. Such attempts lead to an approach based on multidimensional analytic residue theory that relies on techniques of analytic continuation in one or several complex variables. Conjecture 1.3 seems harder to deal with since it fits so well with the search for explicit division formulas in $\widehat{\mathcal{E}^{\prime}\left(\mathbb{R}^{n}\right)}$ that resolve Ehrenpreis's fundamental principle as studied in [36]. (See also [18] or, more recently, [2]). What is known as Ehrenpreis-Montgomery conjecture is the particular case of Conjecture 1.2, when $n=1$. Thanks to Ritt's theorem [52], Conjecture 1.2 in the case $n=1$ reduces to the following.

Conjecture 1.4 (Ehrenpreis-Montgomery conjecture) Let

$$
f(z)=\sum_{k=0}^{M} b_{k}(z) e^{i \alpha_{k} z}, b_{k} \in \overline{\mathbb{Q}}[X], \alpha_{k} \in \overline{\mathbb{Q}} \cap \mathbb{R}
$$

be an exponential polynomial with both algebraic coefficients and frequencies. Then, there are constant $c, A>0, p \in \mathbb{N}$ (depending on $f$ ) such that

$$
\left(f(z)=f\left(z^{\prime}\right)=0 \text { and } z \neq z^{\prime}\right) \Longrightarrow\left|z-z^{\prime}\right| \geq c \frac{e^{-A|\operatorname{Im} z|}}{(1+|z|)^{p}} .
$$

A possible reason for the terminology is the relation between Conjecture 1.4 and the following conjecture by H. Shapiro (1958) mentioned by H.L. Montgomery in a colloquium in Number Theory (Bolyai Janos ed.), see [57, 58]. 
Conjecture 1.5 (Montgomery-Shapiro conjecture) Let $f, g$ be two exponential polynomials that have an infinite number of common zeroes. Then, there is an exponential polynomial $h$ that divides both $f$ and $g$ and has also an infinite number of zeroes.

Unfortunately, I failed to find a precise reference in H. L. Montgomery's work. There seems to be an oral contribution by H. L. Montgomery linking Conjecture 1.4 and Conjecture 1.5. In 1973, Carlo Moreno, under the supervision of L. Ehrenpreis, quoted in the introduction of [48] an unpublished manuscript [47], which should be devoted to his work towards such a conjecture. His thesis (New York, 1971) was centered around it. The idea there was to prove Conjecture 1.4 for sums of exponentials (that is $b_{k} \in \overline{\mathbb{Q}}$ for any $k$ ), involving only a small number of exponential monomials. This is fundamentally different from the methods that arose later (see e.g. [16]), which depend on the rank of the subgroup $\Gamma(f)$ of the real line generated by the frequencies $\alpha_{k}$.

\section{What is known in connection with results in transcendental number theory}

As mentioned in section 1, besides the approach by C. Moreno in his thesis, most of the attempts towards Conjecture 1.4 rely on an additional hypothesis on the rank of the additive subgroup $\Gamma(f)$ of $\overline{\mathbb{Q}} \cap \mathbb{R}$ generated by the frequencies $\alpha_{0}, \ldots, \alpha_{M}$, not on the number of monomials $e^{i \alpha_{k} z}$ involved.

An easy case when Conjecture 1.4 holds is the case where the rank of $\Gamma(f)$ equals 2 , and the $b_{k}$ are constant [39]. The result means in that case that the analytic transcendental curve

$$
t \in \mathbb{C} \mapsto\left(e^{i t}, e^{i \gamma_{1} t}\right), \gamma_{1} \in(\overline{\mathbb{Q}} \cap \mathbb{R}) \backslash \mathbb{Q},
$$

cannot approach a finite subset in $\overline{\mathbb{Q}}^{2}$. Explicitly, any finite linear combination of logarithms of $r$ algebraic numbers $\left(r=3\right.$ here) $\alpha_{\iota}$ with degrees at most $D$, logarithmic heights at most $h$, and with integer coefficients $\nu_{\iota}$ having absolute values less than $B$ is either 0 or bounded from below in absolute value,

$$
\left|\sum_{\iota=1}^{r} \nu_{\iota} \log \alpha_{\iota}\right| \geq \frac{1}{B^{c(r) \times D^{r+2} \log D \times h^{r}}} .
$$

This is a well known fact originally due to A. Baker, see e.g. $[9,10]$ or ([59], section $4)$, for up-to-date results, references or conjectures. When the coefficients $\nu_{\iota}$ are 
algebraic, with heights less than $B$, the following less explicit estimate continues to hold.

$$
\left|\sum_{\iota=1}^{r} \nu_{\iota} \log \alpha_{\iota}\right| \geq \frac{1}{B^{c(r, D) \times h^{\kappa(r)}}}
$$

for some constants $c(r, D)$ and $\kappa(r), D$ being the maximum of the degrees of the $\alpha_{\iota}$ and $\nu_{\iota}$.

The next natural step would be to show that, if $\gamma_{1}, \gamma_{2}$ are two real algebraic numbers such that $\left(1, \gamma_{1}, \gamma_{2}\right)$ are $\mathbb{Q}$-linearly independent, the transcendental curve

$$
t \in \mathbb{C} \mapsto\left(e^{i t}, e^{i \gamma_{1} t}, e^{i \gamma_{2} t}\right)
$$

cannot approach an algebraic curve in $\mathbb{C}^{3}$ which is defined over $\overline{\mathbb{Q}}$; That is, the set of common zeroes of polynomials belonging to $\overline{\mathbb{Q}}\left[X_{1}, X_{2}, X_{3}\right]$. Here we are close to a quantified version of the so-called Schanuel's conjecture (see [59], section 4, for conjectures respect to its quantitative versions).

Conjecture 2.1 (Schanuel's conjecture, "numerical" version) Given s complex numbers $y_{1}, \ldots, y_{s}$ which are $\overline{\mathbb{Q}}$-linearly independent, the transcendence degree of the algebraic extension $\overline{\mathbb{Q}}\left[y_{1}, \ldots, y_{s}, e^{y_{1}}, \ldots, e^{y_{s}}\right]$ over $\overline{\mathbb{Q}}$ is at least equal to $s$.

For $s=1$, this is Gel'fond-Schneider's theorem. The $s=2$ case would imply for example the algebraic independence over $\mathbb{Q}$ of the pair of numbers $(e, \pi)$ or $(\log 2, \log 3)$, and is of course still open. When $\gamma$ is an algebraic number with degree $D \geq 2$ and $\zeta$ a complex number such that $e^{i \zeta} \neq 1$, a result by G. Diaz [34] asserts that, among the exponentials $e^{i \gamma \zeta}, \ldots, e^{i \gamma^{D-1} \zeta}$, at least $[(d+1) / 2]$ are algebraically independent over $\mathbb{Q}$. This result covers Gel'fond's well known result $(D=3)$ and even leads to a quantitative version of it. In fact, the quantitative formulation obtained by D. Brownawell in [14] for $D=3$ (using Gel'fond-Schneider's method) implies the following (rather weak) result respect to Conjecture 1.4, when the rank of $\Gamma(f)$ equals 3 .

Proposition 2.1 ([15]) If $f$ is an exponential sum in one variable with $b_{k} \in \overline{\mathbb{Q}}$ and $\Gamma(f)=\mathbb{Z} \oplus \gamma \mathbb{Z} \oplus \gamma^{2} \mathbb{Z}, \gamma$ being an irrational cubic, then, for any $\epsilon>0$, there is $c_{\epsilon}>0$ depending on $f$ such that

$$
\left(f(z)=f\left(z^{\prime}\right)=0 \text { and } z \neq z^{\prime}\right) \Longrightarrow\left|z-z^{\prime}\right| \geq c_{\epsilon} e^{-|z|^{4+\epsilon}}
$$

The methods introduced by Guy Diaz in [34] in fact allow one to replace $4+\epsilon$ by $1+\epsilon$ in (2.8). In any case, we are indeed very far from what would be the formulation of Conjecture 1.4 in the particular case where $b_{k}$ are constant and the algebraic frequencies belong to the group $\mathbb{Z} \oplus \gamma \mathbb{Z} \oplus \gamma^{2} \mathbb{Z}, \gamma$ being an irrational cubic. 
This is inherent to the approach of the problem via classical methods in diophantine approximation.

Besides these cases and the results of C. Moreno in his unpublished 1971 thesis when the number of monomial terms is small, to my knowledge nothing is really known about Conjecture 1.4, at least in connexion with an approach based on transcendental number theory methods. For some up-to-date survey about Schanuel's conjecture and its quantitative versions, we refer the reader to ([59], sections 3.1 and 4.3).

\section{Using the formal counterpart of Schanuel's nu- merical conjecture}

The point of view I developed with C. A. Berenstein in [16] and section 2 of [15] relies on the fact that the formal analog of Schanuel's conjecture holds, despite the fact that very is known about the numerical Schanuel conjecture. This is a result by J. Ax and B. Coleman [6, 31], following the ideas developped by C. Chabauty [28] and E. Kolchin [40], see also [22] for a modern up-to-date presentation. Here is a formulation.

Theorem 3.1 (Schanuel's conjecture, formal version) Let $y_{1}, \ldots, y_{s}$ be $s$ formal power series in $\mathbb{C}\left[\left[t_{1}, \ldots, t_{k}\right]\right](k \geq 1)$, and $I$ an ideal in $\mathbb{C}\left[X_{1}, \ldots, X_{s}, Y_{1}, \ldots, Y_{s}\right]$, defining in $\mathbb{C}^{2 s}$ an algebraic subvariety $\mathcal{V}(I)$ with dimension less or equal to s, such that

$$
\forall P \in I, P\left(y_{1}(t), \ldots, y_{s}(t), e^{y_{1}(t)}, \ldots, e^{y_{s}(t)}\right) \equiv 0 .
$$

Then, there are rational numbers $r_{1}, \ldots, r_{s}$ and a complex number ${ }^{2} \gamma \in \mathbb{C}$ such that

$$
\sum_{j=1}^{s} r_{j} y_{j}(t) \equiv \gamma
$$

Here is a corollary of the last Theorem that shows the crucial role it plays when studying the slowly decreasing conditions introduced by Ehrenpreis ( e.g. [36]) for ideals generated by exponential polynomials with frequencies in $(i \mathbb{Z})^{n}$. We ignore for the moment any condition of arithmetic type on the coefficients.

\footnotetext{
${ }^{2}$ Unfortunately, even when one specifies arithmetic conditions on the ideal $I$, such as the generating polynomials have algebraic coefficients, nothing more precise can be asserted about the constant $\gamma$. Indeed, this is the main stumbling block to such a result being an efficient tool in proving Conjecture 1.2 or even Conjecture 1.4 .
} 
Corollary 3.1 ([16], Proposition 6.4 and Corollary 6.7) Let $P_{1}, \ldots, P_{N}$ be $N$ polynomials in the $2 n$ variables $\left(X_{1}, \ldots, X_{n}, Y_{1}, \ldots, Y_{n}\right)$, defining an algebraic variety $\mathcal{V}(P)$ in $\mathbb{C}_{z, w}^{2 n}$. Let $\pi_{z}:(z, w) \in \mathbb{C}^{2 n} \mapsto z$ be the projection on the factor $\mathbb{C}_{z}^{n}$. Let $W \subset \mathbb{C}_{z}^{n}$ be the subset defined by

$$
\left(z_{1}, \ldots, z_{n}\right) \notin W \Longrightarrow \operatorname{dim}\left(\mathcal{V}(P) \cap \pi^{-1}(z)\right)=0 \text { or }-\infty .
$$

Then, any irreducible component with strictly positive dimension of the analytic (transcendental) subset

$$
V(F)=\left\{z \in \mathbb{C}^{n} ; F_{j}(z)=P_{j}\left(z_{1}, \ldots, z_{n}, e^{i z_{1}}, \ldots, e^{i z_{n}}\right)=0, j=1, \ldots, N\right\}
$$

lies in $\bar{W}$. In particular, when $N \geq n$, any irreducible component with strictly positive dimension of $V(F)$ lies in the closure in $\mathbb{C}^{n}$ of the set $W^{\prime} \subset \mathbb{C}_{z}^{n}$ defined as

$$
z \notin W^{\prime} \Longrightarrow \operatorname{rank}\left[\left(\frac{\partial P_{j}(z, w)}{\partial w_{k}}\right)_{\substack{1 \leq j \leq N \\ k \leq 1 \leq n}}\right]=n \quad \forall w \in \mathbb{C}^{n} .
$$

The formal analog of Schanuel's conjecture also allows one to give refined versions of Ritt's theorem in several variables such as those formulated in [8]. Here is an example.

Corollary $3.2([16]$, see also [53]) Let

$$
F\left(z_{1}, \ldots, z_{n}\right)=\sum_{k=0}^{M} b_{k}(z) e^{i\left\langle\alpha_{k}, z\right\rangle}
$$

be an exponential polynomial in $n$ complex variables which is identically zero on an algebraic irreducible curve $\mathcal{C}$. Then either all polynomial factors $b_{k}$ vanish identically on $\mathcal{C}$ or else $\mathcal{C}$ is contained in some affine subspace $\left\langle\alpha_{k_{1}}-\alpha_{k_{2}}, z\right\rangle=\gamma$, where $\gamma$ is a complex constant ${ }^{3}$ and $\alpha_{k_{1}} \neq \alpha_{k_{2}}$. If an irreducible polynomial $P \in \mathbb{C}\left[X_{1}, \ldots, X_{n}\right]$ divides $F$ (as an entire function) without dividing all the $b_{k}$, then $P$ is necessarily of the form

$$
P(X)=\left\langle\alpha_{k_{1}}-\alpha_{k_{2}}, X\right\rangle-\gamma .
$$

The main reason such analytic techniques arising from the formal analog of Schanuel's conjecture fail to imply Conjecture 1.2 (or more specifically Conjecture 1.4), is because they do not allow one to keep track of the arithmetic constraints. Though such a goal can be (partially) achieved when adapting Nother Normalization's lemma to the frame of exponential polynomials $P\left(X_{1}, \ldots, X_{n}, e^{Y_{1}}, \ldots, e^{Y_{n}}\right)$ (as in Proposition 6.3 in [16]), it still seems far from providing enough information to make significant advances towards Conjectures 1.2 or 1.4.

\footnotetext{
${ }^{3}$ Here again, additional arithmetic information on $F$ does not impose any arithmetic constraint on $\gamma$.
} 


\section{Arithmetic rigidity and the $\mathcal{D}$-module approach}

\subsection{Lindemann-Weierstrass theorem versus Ritt's factoriza- tion}

The ubiquity that was pointed out in $[4,5]$ with respect to the well known LindemannWeierstrass theorem suggests how arithmetic rigidity is reflected in Ritt's factorization of exponential sums in the one variable setting. Let us recall the classical "numerical" formulation of Lindemann-Weierstrass theorem.

\section{Theorem 4.1 (Lindemann-Weierstrass, "numerical" formulation)}

Let $\alpha_{1}, \ldots, \alpha_{s}$ be s algebraic numbers which are $\mathbb{Q}$-linearly independent. Then their exponentials $e^{\alpha_{1}}, \ldots, e^{\alpha_{s}}$ are algebraically independent over $\overline{\mathbb{Q}}$.

Here is its equivalent "functional" formulation, which appears to be an arithmetic version of Ritt's factorization theorem. In this situation, arithmetic conditions indeed impose drastic rigidity constraints.

\section{Theorem 4.2 (Lindemann-Weierstrass, "functional formulation")}

Let $\mathfrak{f}$ be a formal power series in $\mathbb{Q}[[X]]$, which corresponds to the Taylor development about the origin of an exponential polynomial $f$ with constant coefficients ${ }^{4}$, such that $f(1)=0$, that is $f$ can be divided by $z-1$ as an entire function. Then the quotient

$$
z \mapsto \frac{\mathfrak{f}(X)}{X-1}
$$

is also the formal power series at the origin of an exponential polynomial with constant coefficients ${ }^{5}$.

\subsection{A first ingredient for the proof of Theorem 4.2 : the notion of "size" for a $X d / d X$-module over $\mathbb{K}(X)$}

One of the major ingredients in the "modern" proof $([4,5])$ of Theorem 4.2 is the notion of "being of finite size" for a $X d / d X$ module over $\mathbb{K}(X)$, where $\mathbb{K}$ is a number field. We keep for the moment to the one variable setting.

Let $\mathbb{K}$ be such a number field, and $\mathcal{M}$ be a $X d / d X$-module over $\mathbb{K}(X)$. Assume $\mathcal{M}$ is such that the $\mathbb{K}(X)$ induced module is free with finite $\operatorname{rank}^{6}$. Thus, $\mathcal{M}$ can be

\footnotetext{
${ }^{4}$ Certainly, the coefficients and frequencies of such an exponential polynomial $f$ are in $\overline{\mathbb{Q}}$.

${ }^{5}$ That is, of course, is identically zero. Nevertheless, it seems better to keep this formulation to view the statement as the effect of arithmetic rigidity constraints in Ritt's factorization theorem.

${ }^{6}$ More generally, one may replace $\mathbb{K}(X)$ by some unitary $\mathbb{K}$-algebra containing $\mathbb{K}(X)$, such as $\mathbb{K}[[X]]$, and introduce then the notion of $X d / d X$-module of finite type over $\mathbb{K}[[X]]$.
} 
represented in terms of a basis $\Upsilon=\left(v_{0}, \ldots, v_{\mu-1}\right)$ with the action of the differential operator $X d / d X$ being represented as

$$
(X d / d X)\left[v_{j}\right]=\sum_{k=0}^{\mu-1} G_{j k}(X)\left[v_{k}\right] .
$$

Taking into account the fact that $\mathbb{K}$ is a number field (and thus the arithmetic rigidity), one can introduce a notion of size $\sigma(\mathcal{M})$ as

$$
\sigma(\mathcal{M})=\limsup _{N \rightarrow \infty} \frac{1}{N} \sum_{v \in \Sigma_{\text {finies }}(K)} \log ^{+} \max _{0 \leq p \leq N}\left\|\frac{G^{(p)}(X)}{p !}\right\|_{v}
$$

where $\Sigma_{\text {finies }}$ denotes the set of non archimedian (conveniently normalized) absolute values on the number field $\mathbb{K}$, and $G^{p}$ is the $(\mu, \mu)$ matrix with entries in $\mathbb{K}(X)$, corresponding to the action of $X^{p}(d / d X)^{p}$, expressed within the basis $\Upsilon$ (see for example [3,33]). The size is in fact independent of the choice of the basis $\Upsilon$. The module $\mathcal{M}$ is said to satisfy the Galochkin condition when its size $\sigma(\mathcal{M})$ is finite.

An important result by G. Chudnovsky [29, 30], one that relies on Siegel's lemma ${ }^{7}$, asserts that, if $A$ is a $(\mu, \mu)$ matrix with coefficients in $\mathbb{K}[X]$ such that the differential system

$$
(d / d X-A)[Y]=0
$$

admits a solution $Y_{0}$ in $(\mathbb{K}[[X]])^{\mu}$ with $\mathbb{K}(X)$-linearly independent components, then the size of the corresponding $X d / d X$-module $\mathcal{M}_{A}$ is bounded from above by $C(\Gamma) h\left(Y_{0}\right)$, where $h\left(Y_{0}\right)$ denotes the maximum of the heights of the coefficients of $Y_{0}$, the height being understood here as the height of a formal power series with coefficients in $\mathbb{K}$ (see [3]). In particular, $\mathcal{M}_{A}$ satisfies the Galochkin condition when the differential system admits a solution with $\mathbb{K}(X)$-linearly independent components, which are all $G$-functions (see [3] for various definitions ${ }^{8}$ of such an arithmetic notion). Note that G. Chudnovsky's theorem has been extended to the several variable context by L. di Vizio in [32].

\footnotetext{
${ }^{7}$ See e.g. [33], Chapter VIII, for a pedestrian presentation and a proof.

${ }^{8}$ To say it briefly, a $G$-function is a formal power series in $\overline{\mathbb{Q}}[[X]]$ which is in the kernel of some element in $\overline{\mathbb{Q}}[X, d / d X]$ and, at the same time, has a finite logarithmic height, when considered as a power series in $\overline{\mathbb{Q}}[[X]]$ (see $[3]$ for the notion of logarithmic height for a power series).
} 


\subsection{A second ingredient for the proof of Theorem 4.2: a theorem by N. Katz}

Here again, one keeps to the one variable context. A differential operator with coefficients in $\mathcal{M}_{\mu, \mu}(\mathbb{C}[X])$

$$
\mathcal{L}=\sum_{1}^{L} A_{l}(X)(d / d X)^{q},
$$

it is called fuschian if all its singularities $a \in \mathbb{C} \cup\{\infty\}$ are regular ones. That is, are such that

$$
\min _{l \geq 1}\left(\operatorname{val}_{a}\left(A_{l}\right)-l\right) \geq \operatorname{val}_{a}\left(A_{L}\right) .
$$

A theorem by N. Katz [44] asserts that any $X d / d X$-module over $\mathbb{K}(X)$ ( $\mathbb{K}$ being a number field) which satisfies Galochkin condition is necessarily fuschian.

This result has also an extension to the context of several variables ([32]). Such an extension can be combined with Chudnovsky's theorem in higher dimension, as formulated in geometric terms also in ([32]).

The proof of Theorem 4.2 ([5]) follows from such a combination between Chudnovsky's and Katz's theorems. It relies on the elementary proof proposed in [23], which bypasses the $p$-adic methods based on the Bézivin-Robba criterion that were previously introduced in [24].

\subsection{The $\mathcal{D}$-modules approach}

Let us start here with a few observations about division questions in multivariate complex analysis. This approach is reminiscent of pseudo-Wiener deconvolution methods that involve as deconvolutors filters with transfer functions

$$
\omega \in \mathbb{R}^{n} \longmapsto \frac{\overline{F_{j}(\omega)}}{\|F(\omega)\|^{2}+\epsilon^{2}},
$$

where the $F_{j}, j=1, \ldots, N$, are the transfer functions of the convolutor filters, and $\epsilon^{2}<<1$ stands here for a signal to noise ratio.

Let $F_{1}, \ldots, F_{N}$ be $N$ elements in the Paley-Wiener algebra $\widehat{\mathcal{E}^{\prime}\left(\mathbb{R}^{n}\right)}$. Consider the holomorphic map $z \mapsto F(z):=\left(F_{1}(z), \ldots, F_{N}(z)\right)$ as an holomorphic section of the trivial bundle $\mathbb{C}^{n} \times \mathbb{C}^{N} \rightarrow \mathbb{C}^{n}$, equipped with its canonical basis. Let

$$
\sigma(z)=\frac{\sum_{j=1}^{N} \overline{F_{j}(z)} \otimes e_{j}}{\|F(z)\|^{2}}, \quad z \in \mathbb{C}^{n} \backslash F^{-1}(0) .
$$


It can be shown that there are bundle-valued currents $P_{F}$ and $R_{F}$ in $\mathbb{C}^{n}$ defined by the formulas

$$
\begin{aligned}
& P_{F}:=\left[\|F(z)\|^{2 \lambda} \sum_{r=1}^{n} \frac{\sigma(z) \wedge(\bar{\partial}[\sigma(z)])^{r-1}}{(2 i \pi)^{r}}\right]_{\lambda=0} \\
& R_{F}:=\left[\bar{\partial}\left[\|F(z)\|^{2 \lambda}\right] \wedge \sum_{r=1}^{n} \frac{\sigma(z) \wedge(\bar{\partial}[\sigma(z)])^{r-1}}{(2 i \pi)^{r}}\right]_{\lambda=0} .
\end{aligned}
$$

That is, one analytically continues the complex parameter $\lambda$ from $\{\operatorname{Re} \lambda>>1\}$ to some half-plane $\{\operatorname{Re} \lambda>-\eta\}$ for some $\eta>0$. Note that $\operatorname{Supp} R_{F} \subset F^{-1}(0)$ and that $P_{F}$ and $R_{F}$ are related by $\left.((2 i \pi)\rfloor_{F}-\bar{\partial}\right) \circ P_{F}=1-R_{F}$, where $\rfloor_{F}$ denotes the interior product with $F$.

In order to justify such a construction, one takes a $\log$ resolution $\pi: \widetilde{\mathbb{C}^{n}} \rightarrow \mathbb{C}^{n}$ for the subvariety $\left\{F_{1}=\cdots=F_{N}=0\right\}$. Such a log resolution factorizes through the normalized blow-up of $\mathbb{C}^{n}$ along the coherent ideal sheaf $\left(F_{1}, \ldots, F_{N}\right) \mathcal{O}_{\mathbb{C}^{n}}$. When $N \leq n$ and $F_{1}, \ldots, F_{N}$ define a complete intersection in $\mathbb{C}^{n}$, the current $R_{F}$ reduces to its $(0, N)$ component, which coincides in this case with the current realized in a neighborhhood of $\bigcup_{1}^{N} F_{j}^{-1}(0)$ as the value at $\lambda_{1}=\cdots=\lambda_{N}=0$ of the analytically continued current-valued holomorphic map

$$
\left(\lambda_{1}, \ldots, \lambda_{N}\right) \in\left\{\operatorname{Re} \lambda_{1}>>1, \ldots, \operatorname{Re} \lambda_{N}>>1\right\} \longmapsto \frac{1}{(2 i \pi)^{N}} \bigwedge_{j=N}^{1} \bar{\partial}\left(\frac{\left|F_{j}\right|^{2 \lambda_{j}}}{F_{j}}\right) .
$$

When $F_{1}, \ldots, F_{N}$ are polynomials (that is Fourier transforms of distributions with support $\{0\}$ ), all distribution coefficients of the current $P_{F}$ belong to $\mathcal{S}^{\prime}\left(\mathbb{C}^{n} \simeq \mathbb{R}^{2 n}\right)$, in which case the ideal $\left(F_{1}, \ldots, F_{N}\right)$ is of course closed in the Paley-Wiener algebra. The current $P_{F}$ is said to have Paley-Wiener growth in $\mathbb{C}^{n}$ if and only if all its distribution coefficients $T$ satisfy the weaker condition

$$
\begin{aligned}
& \exists p \in \mathbb{N},, \exists A>0, \exists C>0, \quad \text { such that } \\
& |\langle T, \varphi\rangle| \leq C \sup _{|\underline{\mid l}|+|\underline{m}| \leq p} \sup _{\mathbb{C}^{n}}\left[(1+\|z\|)^{p} e^{A\|\operatorname{Im} z\|}\left|\frac{\partial^{\underline{l}+\underline{m}}[\varphi]}{\partial \zeta \underline{\underline{l}} \partial \bar{\zeta}^{m}}(z)\right|\right] .
\end{aligned}
$$

If $P_{F}$ has Paley-Wiener growth, so has $R_{F}$, since $\left.((2 i \pi)\rfloor_{F}-\bar{\partial}\right) \circ P_{F}=1-R_{F}$. Division methods such as developped in $[19,20,1,2]$, show that, if $P_{F}$ (hence $R_{F}$ ) has Paley-Wiener growth in $\mathbb{C}^{n}$,

$$
\left(\left[I\left(F_{1}, \ldots, F_{N}\right)\right]_{\text {loc }}\right)^{\min (n, N)} \subset I\left(F_{1}, \ldots, F_{N}\right) .
$$

In the particular case where $N \leq n$ and $\left(F_{1}, \ldots, F_{N}\right)$ define a complete intersection in $\mathbb{C}^{n}$, the fact that $P_{F}$ (hence $R_{F}$ ) has Paley-Wiener growth in $\mathbb{C}^{n}$ implies that 
$I\left(F_{1}, \ldots, F_{N}\right)$ is closed in the Paley-Wiener algebra (one can replace the exponent $\min (n, N)$ by 1 in $(4.15))$. When $\left(F_{1}, \ldots, F_{N}\right)$ have no common zeroes in $\mathbb{C}^{n}$, it is therefore equivalent to say that $I\left(F_{1}, \ldots, F_{N}\right)$ is closed in the Paley-Wiener algebra or to say that $P_{F}$ has Paley-Wiener growth (here $R_{F} \equiv 0$ since $\left.F^{-1}(0)=\emptyset\right)$. Conjecture 1.2 suggests then the following conjecture.

Conjecture 4.1 Let $F_{1}, \ldots, F_{N}$ be $N$ exponential polynomials such as in Conjecture 1.2. The current $P_{F}$ (hence also $R_{F}$ ) has Paley-Wiener growth .

Remark 4.1 Conjecture 4.1 implies Conjecture 1.4: when $n=1$, take $N$ large enough and $F_{1}, \ldots, F_{N}$ the list of successive derivatives of the exponential polynomial $f: z \mapsto \sum_{k=0}^{M} b_{k}(z) e^{i \alpha_{k} z}$ (see e.g. [15]).

In order to rephrase Conjecture 4.1 in more algebraic terms, let us recall the following trick. If $\operatorname{Re} \beta>0$, and $t_{1}, \ldots, t_{N}$ are $N$ strictly positive numbers, then one has, for any $\left.\left(\gamma_{1}, \ldots, \gamma_{N-1}\right) \in\right] 0, \infty\left[{ }^{N-1}\right.$ such that $\gamma_{1}+\cdots+\gamma_{N-1}<\operatorname{Re} \beta$,

$$
\begin{aligned}
& \left(t_{1}+\cdots+t_{N}\right)^{-\beta} \\
& =\frac{1}{(2 i \pi)^{N-1} \Gamma(\beta)} \int_{\gamma_{1}+i \mathbb{R}} \cdots \int_{\gamma_{N-1}+i \mathbb{R}} \Gamma_{N}^{*}(\zeta) t_{1}^{-\zeta_{1}} \cdots t_{N-1}^{-\zeta_{N-1}} t_{N}^{\zeta^{*}} d \zeta_{1} \cdots d \zeta_{N-1},
\end{aligned}
$$

where

$$
\Gamma_{N}^{*}(\zeta)=\Gamma\left(\zeta_{1}\right) \cdots \Gamma\left(\zeta_{N-1}\right) \Gamma\left(\beta-\zeta_{1}-\cdots-\zeta_{N-1}\right), \zeta^{*}=\sum_{k=1}^{N-1} \zeta_{k}-\beta .
$$

Formula (4.16) allows the transformation of the additive operation between the $t_{j}$ (namely $\left(t_{1}+\cdots+t_{N}\right)^{-\beta}$ ) into a multiplicative one (namely $t_{1}^{-\zeta_{1}} \cdots t_{N-1}^{-\zeta_{N-1}} t_{N}^{\zeta^{*}}$, once in the integrand). One can view it as a continuous version of the binomial formula (with negative exponent). Taking for example $t_{j}=\left|F_{j}(z)\right|^{2}, j=1, \ldots, N$, it follows that one way then to tackle Conjecture 4.1 could be to study (first formally, then numerically in $\mathbb{C}^{n}$, pairing antiholomorphic coordinates with holomorphic ones in order to recover positivity) the analytic continuation of

$$
\lambda=\left(\lambda_{1}, \ldots, \lambda_{N}\right) \longmapsto \prod_{j=1}^{N}\left(F_{j}\left(z_{1}, \ldots, z_{n}\right)\right)^{\lambda_{j}} .
$$

When $F_{1}, \ldots, F_{N}$ are polynomials in $\mathbb{K}\left[X_{1}, \ldots, X_{n}\right]=\mathbb{K}[X]$, where $\mathbb{K}$ is a number field, one may consider the $\mathbb{K}(\lambda)\langle X, d / d X\rangle$-module $\mathfrak{M}(F)$ freely generated by a single generator (formally denoted as $\mathfrak{F}^{\lambda}=\mathfrak{F}_{1}^{\lambda_{1}} \otimes \cdots \otimes \mathfrak{F}_{N}^{\lambda_{N}}$ ), namely

$$
\mathfrak{M}(F)=\mathbb{K}(\lambda)[X]\left[\frac{1}{F_{1}}, \ldots, \frac{1}{F_{N}}\right] \cdot \mathfrak{F}^{\lambda} .
$$


This $\mathbb{K}(\lambda)\langle X, d / d X\rangle$-module is holonomic (i.e. $\operatorname{dim} \mathfrak{M}(F)=n$ ). A noetheriannity argument (see e.g. [35]) implies then that there exists a set of global Bernstein-Sato algebraic relations

$$
\mathcal{Q}_{j}(\lambda, X, d / d X)\left[F_{j} \cdot \mathfrak{F}^{\lambda}\right]=\mathcal{B}(\lambda) \cdot \mathfrak{F}^{\lambda}, j=1, \ldots, N,
$$

where $\mathcal{B} \in \mathbb{K}[\lambda]$ and $\mathcal{Q}_{j} \in \mathbb{K}[\lambda]\langle X, d / d X\rangle, j=1, \ldots, N$. Such a set of algebraic relations (4.18) can be used in order to express (via (4.16) with $t_{j}=\left|F_{j}(z)\right|^{2}, t_{=} 1, \ldots, N$ ) the current $P_{F}$ as a current with coefficients in $\mathcal{S}^{\prime}\left(\mathbb{C}^{n}\right)$.

Local analytic analogs of global Bernstein-Sato algebraic relations (4.18) indeed exist. When $f_{1}, \ldots, f_{N}$ are $N$ elements in $\mathcal{O}_{\mathbb{C}^{n}, 0}$ and $\mathfrak{t}$ is an holonomic distribution about the origin in $\mathbb{C}^{n}$ (for example, a distribution coefficient of some integration current $[V]$, or of some Coleff-Herrera current, see [27]), then there exists a set of local Bernstein-Sato analytic equations

$$
q_{\mathfrak{t}, j}(\lambda, \zeta, \partial / \partial \zeta)\left[f_{j} \cdot \mathfrak{f}^{\lambda} \otimes \mathfrak{t}\right]=b_{\mathfrak{t}}(\lambda) \cdot \mathfrak{f}^{\lambda} \otimes \mathfrak{t}, j=1, \ldots, N,
$$

where $q_{\mathfrak{t}, j}$ denotes a germ at the origin of a holomorphic differential operator with coefficients analytic in $\zeta$ and polynomial in $\lambda$, and $b_{\mathfrak{t}}$ is a finite product of affine forms $\kappa_{0}+\kappa_{1} \lambda_{1}+\cdots+\kappa_{n} \lambda_{n}$, with $\kappa_{0} \in \mathbb{N}^{*},\left(\kappa_{1}, \ldots, \kappa_{M}\right) \in \mathbb{N}^{M} \backslash\{0\}([25,55,42,26])$. Unfortunately, such a local result does not provide any algebraic information about the $q_{\mathbf{t}, j}$, when for example the $f_{j}$ 's represent the germs at the origin of exponential polynomials of the form (1.3), as in Conjecture 1.2 or Conjecture 1.3.

One intermediate way to proceed in this case is to consider the case of formal power series. For example, let us suggest an approach to tackle Conjecture 1.4 for exponential sums. Consider an exponential sum

$$
f: \zeta \in \mathbb{C} \longmapsto \sum_{k=0}^{M} b_{k} e^{i \alpha_{k} \zeta},
$$

with algebraic coefficients $b_{k}$, and purely imaginary algebraic distinct frequencies $i \alpha_{k}$. Let $\mathbb{K}$ be the number field generated by the $b_{k}$ 's, the $\alpha_{k}$ 's, and $i$. Let $n \geq 1$ be the rank of the subgroup $\Gamma(f)=\mathbb{Z} \alpha_{0}+\cdots+\mathbb{Z} \alpha_{M}$, and $\left(\gamma_{1}, \ldots, \gamma_{n}\right)$ be a basis of $\Gamma(f)$. For each $j=1, \ldots, M$, let $P_{j} \in \mathbb{K}\left[X_{1}, \ldots, X_{n}\right]$ such that

$$
\frac{d^{j-1} f}{d \zeta^{j-1}}(z)=P_{j}\left(e^{i \gamma_{1} z}, \ldots, e^{i \gamma_{n} z}\right), \quad \forall z \in \mathbb{C},
$$

and $P:=\left(P_{1}, \ldots, P_{M}\right): \mathbb{C}^{n} \rightarrow \mathbb{C}^{M}$. Let $N=M+n-1$, and the exponential polynomials $F_{1}, \ldots, F_{N}$ be defined as follows:

- for $j=1, \ldots, M, F_{j}$ is the exponential sum in $n$ variables, with coefficients in $\mathbb{K}$

$$
\left(z_{1}, \ldots, z_{n}\right) \longmapsto F_{j}(z)=P_{j}\left(e^{i z_{1}}, \ldots, e^{i z_{n}}\right)
$$


- for $j=1, \ldots, n-1, F_{M+j}$ is the linear form, also with coefficients in $\mathbb{K}$,

$$
\left(z_{1}, \ldots, z_{n}\right) \longmapsto \gamma_{n} z_{j}-\gamma_{j} z_{n} .
$$

Let $\xi$ be a point in $\mathbb{C}^{n}$, such that $e^{i \xi} \in \mathbb{K}^{n} \cap\{P=0\}$. The Taylor developments of $F_{1}, \ldots, F_{M}$ at $\xi$ correspond to power series $\mathfrak{f}_{1, \xi}, \ldots, \mathfrak{f}_{M, \xi}$ in $\mathbb{K}\left[\left[X_{1}, \ldots, X_{n}\right]\right]$, while the Taylor developpements at $\xi$ of $F_{M+1}, \ldots, F_{N}$ correspond to the affine power series

$$
\mathfrak{f}_{M+j, \xi}: X=\left(X_{1}, \ldots, X_{n}\right) \longmapsto \mathfrak{u}_{j}+\left(\gamma_{n} X_{j}-\gamma_{j} X_{n}\right), j=1, \ldots, n-1,
$$

where $\mathfrak{u}_{j}=\gamma_{n} \xi_{j}-\gamma_{j} \xi_{n}$ is a linear combination of logarithms of algebraic numbers with algebraic coefficients. Here $\mathfrak{u}_{1}, \ldots, \mathfrak{u}_{n-1}$ can be interpreted as parameters. Inspired by [11], one could conjecture ${ }^{9}$ the existence of a set of global formal generic Bernstein-Sato relations:

$$
\mathfrak{Q}_{\xi, j}\left(\lambda, X, \mathfrak{u}_{1}, \ldots, \mathfrak{u}_{n-1}, d / d X\right)\left[\mathfrak{f}_{j, \xi} \cdot \mathfrak{F}_{\xi}^{\lambda}\right]=\mathfrak{g}_{\xi}\left(\mathfrak{u}_{1}, \ldots, \mathfrak{u}_{n-1}\right) \mathfrak{b}_{\xi}(\lambda) \cdot \mathfrak{F}_{\xi}^{\lambda}, j=1, \ldots, N,
$$

where $\mathfrak{F}_{\xi}^{\lambda}=\mathfrak{f}_{1, \xi}^{\lambda_{1}} \otimes \ldots \mathfrak{f}_{N, \xi}^{\lambda_{N}}, \mathfrak{Q}_{\xi, j}$ is a differential operator with coefficients in $\mathbb{K}[\lambda][[\mathfrak{u}, X]]$, $\mathfrak{g}_{\xi} \in \mathbb{K}[[\mathfrak{u}]], \mathfrak{b}_{\xi} \in \mathbb{K}[\lambda]$. Moreover, an argument based on Siegel's method (and principle), as that developed by L. Ehrenpreis ${ }^{10}$ in [37], could be then used in order to ensure then that the formal power series coefficients (in $X, \mathfrak{u}$ ) of the $\mathfrak{Q}_{j}$ (considered as polynomials in $\lambda$ and $d / d X$ ) have indeed a radius of convergence which is bounded from below by $\rho>0$, independently of $\xi$, provided $e^{i \xi}$ belongs to a compact subset of $\left(\mathbb{C}^{*}\right)^{n}$. Then (4.20) would provide a semi-global Bernstein-Sato set of relations. The results quoted in section 4, which rely on Siegel's lemma (see e.g. the proof of Chudnovsky's theorem in [33], or the approach to Gelfand-Shidlovsky theorem as in [21]) give indeed some credit to the conjectural existence of such a collection (indexed by $\xi$, with $e^{i \xi} \in \mathbb{K}^{n} \cap P^{-1}(0)$ ) of Berntein-Sato sets of semi-global relations $\mathcal{B}_{\xi}$ as (4.20). One could then identify terms with lower degree in $\mathfrak{u}$ in (4.20) and thus assume, in each set of relations $\mathcal{B}_{\xi}$ such as $(4.20)$, that $\mathfrak{g}_{\xi}$ is homogeneous in $\mathfrak{u}$. In the particular case $n=3$ (where we recall almost nothing is known concerning Conjecture 1.4, see section 2), one could thus assume that $\mathfrak{g}_{\xi}$ factorizes as a product of linear factors $\beta_{\xi, 1} \mathfrak{u}_{1}+\beta_{\xi, 2} \mathfrak{u}_{2}$, where $\beta_{\xi, 1}$ and $\beta_{\xi, 2}$ belong to $\mathbb{K}$. Combining this with A. Baker's theorem $\left(\operatorname{take}\left(\mathfrak{u}_{1}, \mathfrak{u}_{2}\right)=\left(\log \xi_{1}+2 i k_{1} \pi, \log \xi_{2}+2 i k_{2} \pi\right),\left(k_{1}, k_{2}\right) \in \mathbb{Z}^{2}\right)$, one would get (with (4.20)) some way to control the analytic continuation procedure (4.17), leading to the conjectural lower estimates

$$
\sum_{j=1}^{M}\left|P_{j}\left(e^{\gamma_{1} z}, \ldots, e^{\gamma_{n} z}\right)\right|=\sum_{1}^{M}\left|\frac{d^{j-1} f}{d \zeta^{j-1}}(z)\right| \geq c \frac{e^{-A|\operatorname{Im} z|}}{(1+|z|)^{p}}
$$

\footnotetext{
${ }^{9}$ The lines which follow intend just to sketch what could be a conjectural approach to Conjecture 1.4 for exponential sums $f$ such that $\Gamma(f)$ has small rank.

${ }^{10}$ Note that this work of L. Ehrenpreis appeared in the Lecture Notes volume where appeared also the important results by G. Chudnovsky $[29,30]$.
} 
that ensure (1.5) (see [15]).

The conjectural approach proposed above can be seen as an attempt to take into account the intrinsic arithmetic rigidity of such problems that the results quoted in section 4 suggest.

Another approach, one that would seem more direct, would be to try to mimic the algebraic construction that leads to the construction of a global set of BernsteinSato relations such as (4.18) when $F_{1}, \ldots, F_{N}$ belong to $\mathbb{K}\left[X_{1}, \ldots, X_{n}\right]$. That is, let $F_{1}, \ldots, F_{N}$ be $N$ exponential polynomials of the form

$$
F_{j}(z)=P_{j}\left(z_{1}, \ldots, z_{n}, e^{i \gamma_{1,1} z_{1}}, \ldots, e^{i \gamma_{1, N_{1}} z_{1}}, \ldots, e^{i \gamma_{n, 1} z_{n}}, \ldots, e^{i \gamma_{n, N_{n}} z_{n}}\right), j=1, \ldots, N,
$$

where $P_{j} \in \mathbb{K}\left[X_{1}, \ldots, X_{n}, Y_{1,1}, \ldots, Y_{1, N_{1}}, \ldots, Y_{n, 1}, \ldots, Y_{n, N}\right]$, the $\gamma_{j, k}$ being also elements in $\mathbb{K}$ such that $\gamma_{j, 1}, \ldots, \gamma_{j, N_{j}}$ are $\mathbb{Q}$-linearly independent for any $j=1, \ldots, n$. Instead of the Weyl algebra $\mathbb{K}(\lambda)\langle X, d / d X\rangle$, one could introduce a non commutative algebra such as

$$
\mathbb{K}\left(\lambda_{1}, \ldots, \lambda_{n}\right)\left\langle X_{1}, \ldots, X_{n}, Y_{1,1}, \ldots, Y_{1, N_{1}}, \ldots, Y_{n, 1}, \ldots, Y_{n, N_{n}}, \partial_{1}, \ldots, \partial_{n}\right\rangle,
$$

with the following commutation rules: for any $j, k \in\{1, \ldots, n\}$, for any $l \in\left\{1, \ldots, N_{j}\right\}$,

$$
\left[\partial_{k}, X_{j}\right]=-\delta_{j k}, \quad\left[X_{k}, Y_{j, l}\right]=0, \quad\left[\partial_{k}, Y_{j, l}\right]=-\gamma_{j, l} \delta_{k l} Y_{j, l} .
$$

One may consider, as in the Weyl algebra case, the $\mathbb{K}(\lambda)\langle X, Y, \partial\rangle$-module

$$
\mathfrak{M}(F)=\mathbb{K}(\lambda)[X, Y, \partial]\left[\frac{1}{F_{1}}, \ldots, \frac{1}{F_{N}}\right] \cdot \mathfrak{F}^{\lambda} .
$$

Nœtheriannity arguments based on the concept of dimension ${ }^{11}$ for such a module lead (inspired by the argument described by F. Ehlers in [35]) to the existence, in some very particular cases, of what would be a substitute for a set of global Bernstein-Sato relations such as (4.18) (see [18]). Unfortunately, the results obtained here cover only situations basically quite close of that of Conjecture 1.4 when $\operatorname{rank} \Gamma(f) \leq 2$. Here are the results obtained that way :

- the current $P_{F}$ attached to any system $F=\left(F_{1}, \ldots, F_{N}\right), F_{j}\left(z_{1}, \ldots, z_{n}\right)=$ $P_{j}\left(z_{1}, \ldots, z_{n}, e^{i z_{n}}\right), j=1, \ldots, N$, where $P_{j} \in \mathbb{C}\left[X_{1}, \ldots, X_{n}, Y\right]$, has Paley-Wiener growth in $\mathbb{C}^{n}$;

- the current $P_{F}$ attached to any system $F=\left(F_{1}, \ldots, F_{N}\right), F_{j}\left(z_{1}, \ldots, z_{n}\right)=$ $P_{j}\left(z_{1}, \ldots, z_{n-1}, e^{i z_{n}}, e^{i \gamma z_{n}}\right), j=1, \ldots, N$, where $P_{j} \in \overline{\mathbb{Q}}\left[X_{1}, \ldots, X_{n-1}, Y_{1}, Y_{2}\right]$ and $\gamma \in(\overline{\mathbb{Q}} \cap \mathbb{R}) \backslash \mathbb{Q}$, has Paley-Wiener growth in $\mathbb{C}^{n}$.

\footnotetext{
${ }^{11}$ That is on concepts of algebraic, not really arithmetic, nature, though arihmetics is deeply involved.
} 
Note that only the second situation carries an arithmetic structure. The methods developed in [18] failed, at least for their intended purpose of making progress towards Conjectures 1.2 or even 1.4. For example, they do not seem to be of any help towards Conjecture 1.4, when $\operatorname{rank}(\Gamma(f))=2$ and $f$ is a true exponential polynomial (not an exponential sum). The main reason for such a failure is that these methods take into account only the concept of dimension, and ignore that of logarithmic size. On the other hand, the conjectural approach towards Conjecture 1.4 when $\operatorname{rank} \Gamma(f)=3$ (such as sketched above) was taking into account such concepts, basically through Siegel's lemma. It is natural to ask the following question: can some argument based on a filtration with respect to the size lead to what would be a substitute for a set of global Bernstein-Sato relations such as (4.18) or (4.20)? That would indeed provide a decisive step towards all conjectures mentioned here.

\section{Some other miscellaneous approaches}

This paper is intended to give brief, up-to-date discussions of the fascinating conjectures arising from arithmetic considerations added to L. Ehrenpreis's contributions to the study of the "slowly decreasing condition" in the Paley-Wiener algebra. One should add that recent developments in amœba theory $[49,50,43]$, in relation with tropical geometry, might also be of some interest for such conjectures. Unfortunately, they usually are more adapted to the case of complex frequencies ${ }^{12}$ than to the most delicate so-called "neutral case" where all frequencies are purely imaginary as in the questions discussed here. The most serious stumbling block is that, from the combinatorics point of view, when dealing with "algebraic" cones in $\mathbb{R}^{n}$, one is missing Gordon's lemma. One needs then to bypass such a difficulty; see, for example, [12] for the construction of toric varieties associated to non rational fans. In this connection, we mention some references that might inspire ideas for deciding such conjectures about exponential sums [38, 43, 41, 45, 46, 49, 50, 51, 54, 56]. Unfortunately, most of them do not really take into account the arithmetic constraints, and are more in the spirit of C. Moreno's papers [47, 48].

\section{References}

[1] M. Andersson, Residue currents and ideals of holomorphic functions, Bull. Sci. Math. 128 (2004), no. 6, pp. 481-512.

[2] M. Andersson, E. Wulcan, Residue currents with prescribed annihilator ideals, Ann. Sci. École Norm. Sup. 40 (2007), pp. 985-1007.

\footnotetext{
${ }^{12}$ Polya's theory, see also [7]
} 
[3] Y. André, G-Functions and Geometry, Aspects of Mathematics, Vieweg, 1989.

[4] Y. André, Séries Gevrey de type arithmétique, I. Théorèmes de pureté et de dualité, Annals of Mathematics 151 (2000), pp. 705-740.

[5] Y. André, Séries Gevrey de type arithmétique, II. Transcendance sans transcendance, Annals of Mathematics 151 (2000), pp. 741-756.

[6] J. Ax, On Schanuel's conjectures, Ann. of Math. 93 (1971), pp. 252-268.

[7] R. Bellman, K. Cooke, Differential difference equations, Academic Press, New York, 1963.

[8] C. A. Berenstein, A. Dostal, The Ritt theorem in several variables, Ark. Math. 12 (1974), pp. 267-280.

[9] A. Baker, Transcendental Number Theory, Cambridge University Press, 1979.

[10] A. Baker, G. Wülstholz, Logarithmic forms and group varieties, J. Reine Angew. Math. 442 (1993), pp. 19 - 62.

[11] R. Bahloul, Global generic Bernstein-Sato polynomial on an irreducible algebraic scheme, Proc. Japan Acad. 79, Ser. A (2003), pp. 146 - 149.

[12] F. Battaglia, E. Prato, Generalized toric varieties for simple non rational convex polytopes, Intern. Math. Res. Notices 24 (2001), pp. 1315-1337.

[13] C. A. Berenstein, R. Gay, Complex analysis and special topics in harmonic analysis, Springer-Verlag, New York, 1995.

[14] D. Brownawell, Pairs of polynomials small at a number to certain algebraic numbers, Séminaire Delange-Pisot-Poitou 11, 1975-1976, pp. 1-12.

[15] C. A. Berenstein, A. Yger, On Lojasiewicz type inequalities for exponential polynomials, J. Math. Anal. Appl. 129 (1988), pp. 166-195.

[16] C. A. Berenstein, A. Yger, Ideals generated by exponential polynomials, Advances in Mathematics 60 (1986), pp. 1-80.

[17] C. A. Berenstein, A. Yger, Exponential polynomials and $\mathcal{D}$-modules, Compositio Mathematica 95 (1995), pp. 131-181.

[18] C. A. Berenstein, A. Yger, Division interpolation methods and Nullstellensätze, pp. 41-59 in Analysis, Geometry, Number Theory: The Mathematics of Leon Ehrenpreis, E. L. Grinberg [et al.] (eds.), Contemporary Mathematics 251, AMS 1999. 
[19] C. A. Berenstein, R. Gay, A. Vidras, A. Yger, Residue currents and Bézout identities, Progress in Mathematics 114, Birkhäuser, 1993.

[20] C. A. Berenstein, A. Vidras, A. Yger, Multidimensional residue theory and applications, manuscript in preparation, AMS Mathematical Surveys and Monographs.

[21] D. Bertrand, On André's proof of the Siegel-Shidlovsky theorem, pp. 51-63 in Colloque Franco-Japonais: Théorie des Nombres Transcendants (Tokyo, 1998), Sem. Math. Sci., 27, Keio Univ., Yokohama, 1999.

[22] D. Bertrand, Théories de Galois différentielles et transcendance, Annales Inst. Fourier 59 (2009), no. 7, pp. 2773-2803.

[23] F. Beukers, J. P. Bézivin, P. Robba, An alternative proof of the LindemannWeierstrass theorem, American Monthly 97 (1990), no. 3, pp. 193-197.

[24] J. P. Bézivin, P. Robba, A new p-adic method for proving irrationality and transcendence results, Annals of Mathematics 129 (1989) pp. 151-160.

[25] J. E. Björk, Rings of of differential operators, North-Holland, Amsterdam, 1979.

[26] J. E. Björk, Analytic D-modules and applications, Mathematics and its Applications 247, Kluwer Academic Publishers Group, Dordrecht, 1993.

[27] J. E. Björk, Residues and D-modules, pp. 605-651 in The legacy of Niels Henrik Abel, O.A. Laudal, R. Piene (eds.), Springer-Verlag, Berlin (2004).

[28] C. Chabauty, Sur les équations diophantiennes liées aux unités d'un corps de nombres algébrique fini, Ann. Math. Pura Appl. 17 (1938), pp. 127-168.

[29] D. Chudnovsky, G. Chudnovsky, Applications of Padé approximations to diophantine inequalities in values of $G$-functions, pp. 9-51 in Number theory (New York 1983-1984), Lecture Notes in Math. 1135, Springer-Verlag, Berlin (1985).

[30] D. Chudnovsky, G. Chudnovsky, Applications of Padé approximation to the Grothendieck conjecture on linear differential equations, pp. 52-100 in Number theory (New York 1983-1984), Lecture Notes in Math. 1135, Springer-Verlag, Berlin (1985).

[31] R. Coleman, A generalization of the Ax-Schanuel theorem, Amer. J. Math. 102 (1980) pp. 595-624.

[32] L. di Vizio, Sur la théorie géométrique des $G$-fonctions, le théorème de Chudnovsky à plusieurs variables, Math. Ann. 319 (2001), pp. 181-213. 
[33] B. Dwork, G. Gerotto, F. J. Sullivan, An introduction to G-functions, Annals of Mathematics Studies 133, Princeton University Press, 1994.

[34] G. Diaz, Grands degrés de transcendance pour des familles d'exponentielles, C.R. Acad. SCi. Paris Sér. I Math. 305 (1987), no. 5, pp. 159-162.

[35] F. Ehlers, The Weyl algebra, pp. 173-206 in Algebraic D-modules (Perspectives in Mathematics), A. Borel, J. Coates, S. Helgason (eds.), Academic Press, Boston, 1987.

[36] L. Ehrenpreis, Fourier Analysis in several complex variables, Wiley, 1970.

[37] L. Ehrenpreis, Transcendental numbers and partial differential equations, pp. 112-125 in Number theory (New York, 1983-1984), Lecture Notes in Math. 1135, Springer-Verlag, Berlin (1985).

[38] M. Forsberg, M. Passare, A. Tsikh, Laurent determinants and arrangements of hyperplane amoebas, Adv. in Maths. 151 (2000), pp. 45-70.

[39] F. Gramain, Solutions indéfiniment dérivables et solutions presque périodiques d'une équation de convolution, Bull. Soc. Math. France 104 (1976), pp. 401-408.

[40] E. Kolchin, Algebraic groups and algebraic dependence, Amer. J. Math. 90, 1968, pp. 1151-1164.

[41] D. Gurevich, Closed ideals with zero dimensional root set in certain rings of holomorphic functions, J. Soviet Math. 9 (1978), pp. 172-182.

[42] A. Gyoja, Bernstein-Sato's polynomial for several analytic functions, J. Math. Kyoto Univ. 33, (1993), no. 2, pp. 399-411.

[43] A. Henriques, An analog of convexity for complements of amœebas of higher codimension, an answer to a question asked by B. Sturmfels, Adv. Geom. 4 (2004), no. 1, pp. 61-73.

[44] N. Katz, Algebraic solutions of Differential Equations ( $p$-curvature and the Hodge filtration), Invent. Math. 18 (1972), pp. 1-118.

[45] B. Ya. Kazarnovskii, On zeros of exponential sums, Dokl. Akad. Nauk SSSR, 257 (1981), no. 4, pp. 804-808.

[46] B. Ya. Kazarnovskii, Exponential analytic sets, Functional Analysis and its applications, 31 (1997), no. 2, pp. 86-94.

[47] C. Moreno, Zeroes of exponential polynomials, 1973 (unpublished). 
[48] C. Moreno, The zeroes of exponential polynomials (I), Compositio Math. 26 (1973), no. 1, 69-78.

[49] M. Passare, H. Rullgård, Amœebas, Monge-Ampère measures, and triangulations of the Newton polytope, Duke Math. J. 121 (2004), no. 3, pp. 481-507.

[50] K. Purbhoo, A Nullstellensatz for amœbas, Duke Mathematical Journal 141 (2008), no. 3, 407-445.

[51] A. Yu. Rashkovskii, Zeros of holomorphic almost periodic mappings with independent components. Complex Variables 44 (2001) 299 - 316.

[52] J. F. Ritt, A factorization theory for functions $\sum_{i=1}^{n} a_{i} e^{\alpha_{i} x}$, Trans. Amer. Math. Soc. 29 (1927), no. 3, pp. 584-596.

[53] L. I. Ronkin, Functions of completely regular growth, Mathematics and its Applications (Soviet Series), 81. Kluwer Academic Publishers, Dordrecht, 1992.

[54] L. I. Ronkin, On zeros of almost periodic functions generated by holomorphic functions in multicircular domain, pp. 243-256 in Complex Analysis in Modern Mathematics, Fazis, Moscow, 2001.

[55] C. Sabbah, Proximité évanescente II. Équations fonctionnelles pour plusieurs fonctions analytiques, Compositio Math 64 (1987), pp. 213-241.

[56] J. Silipo, Amibes de sommes d'exponentielles, Canadian J. Math. 60, 1 (2008), pp. 222-240.

[57] R. Tijdeman, A. Van der Poorten, On common zeroes of exponential polynomials, Enseignement Mathématique 21 (1975), no. 2, pp. 57-67.

[58] A. Van der Poorten, A note on the zeroes of exponential polynomials, Compositio Math. 31 (1975), no. 2, pp. 109-113.

[59] M. Waldschmidt, Open diophantine problems, Mosc. Math. J. 4, no. 1 (2004), pp. 245-305. 